\title{
Der Nachlass von Hans Georg Bertram
}

Dass Hans Georg Bertram Spuren in der evangelischen Kirchenmusik hinterlassen würde, zeichnete sich früh ab - dass sein Nachlass aber in der Württembergischen Landesbibliothek landen würde, ergab sich erst durch spätere Lebensabschnitte.

Bertram wurde 1936 in Gießen an der Lahn geboren. Sein Vater war Professor für evangelische Theologie und ordinierter Pfarrer, die Mutter als Säuglingskrankenschwester sozial engagiert. Vor allem mit seinen vier Geschwistern und seiner Mutter musizierte Bertram schon als Kind. Früh erhielt er ersten Instrumentalunterricht, zuerst auf der Blockflöte, bald folgten Klavier und Geige. Auch im Schulorchester engagierte sich Bertram aktiv, hier trat er unter anderem als Vokalsolist auf. Im Alter von 13 Jahren begann Bertram schließlich auch, Orgel zu spielen und konnte bereits ein Jahr später seine erste nebenamtliche Organistenstelle in einem Nachbarort antreten. 1953 wechselte Bertram als Organist und Chorleiter in die Petrusgemeinde in Gießen. Etwa Mitte der 1950er Jahre entstanden zudem seine ersten Kompositionen. ${ }^{1}$

Zum Studium der Evangelischen Kirchenmusik und der Komposition kam Bertram 1956 nach Stuttgart. Besonders prägend waren für inn seine Lehrer Hans-Arnold Metzger und Johann Nepomuk David. Parallel zur Kirchenmusik studierte Bertram an den Universitäten Tübingen und Würzburg Musikwissenschaft und Klassische Philologie. ${ }^{2}$ Beide Studien vereinte er in seiner Dissertation über das musikalische Schaffen seines Lehrers David. ${ }^{3}$ Nach seinem Studium ging Bertram 1963 zunächst zurück an die Petruskirche in Gießen. 1966 heiratete er Hanna Müller, mit der er sechs Kinder bekam. Nach 15 Jahren Dienst an der Petruskirche erfolgte im Herbst 1978 ein Umbruch: Die Familie zog nach Esslingen, da Bertram als Dozent für Orgelspiel an die dortige Kirchenmusikschule berufen worden war. ${ }^{4}$ Gleichzeitig trat Bertram die Bezirkskantorenstelle an der Esslinger Stadtkirche St. Dionys an und war hier unter anderem auch mitverantwortlich für die Gestaltung der "Stunde der Kirchenmusik". Die Dozententätigkeit hatte Bertram bis zum Wintersemester 1997/98 inne, die Kantorenstelle an St. Dionys sogar noch darüber hinaus. ${ }^{5}$ Für sein Wirken erhielt Bertram zahlreiche Ehrungen, unter anderem wurde er 1992 mit dem Esslinger Kulturpreis ausgezeichnet. ${ }^{6}$

Bertram verstarb 2013 in Berlin. Als Aufbewahrungsort für seinen Nachlass hatte Bertram selbst die WLB gewählt. Ein Teil seiner Manuskripte kam bereits als Vorlass ins Haus; der Rest wurde nach seinem Tod von seiner Witwe Hanna Bertram nachgeliefert, sodass sich der umfangreiche Nachlass unter der Signatur Cod. hist. qt. 818 nun vollständig in der WLB befindet. Bertram selbst äußerte zu Lebzeiten den Wunsch, sein Nachlass möge zugänglich bleiben und von Interessierten benutzt werden können.

Der Nachlass Bertrams umfasst in erster Linie einzigartige Materialien zu seinem kompositorischen Schaffen: Manuskripte, Kopien mit handschriftlichen Eintragungen, persönliche Spielexemplare, Analysehinweise und Fehlerlisten, aber auch gedruckte Ausgaben seiner Werke. Neben Kompositionen finden sich auch Texte, Korrespondenz, Tonträger und Dokumente. Die Kompositionen stellen aber den interessantesten und umfangreichsten Teil des Nachlasses dar und sind wiederum unterteilt in Bühnenwerke, Vokalmusik, Instrumentalmusik, Bearbeitungen/Fremde Werke, Skizzen/Fragmente und Varia. Die Bühnenwerke und die Vokalmusik waren bereits 2016 von Erdmute Schruhl im Rahmen eines Praktikums detailliert in einer hausinternen Datei erschlossen und archivgerecht verpackt worden. Auch die Programmhefte aus dem Bereich der Dokumente waren schon von Praktikanten erschlossen worden. Der Rest des Nachlasses lag zu Beginn

1) Haist, Susanne: Hans Georg Bertram. Komponist und Kirchenmusiker. Frankfurt am Main [u. a.] 1999 (Europäische Hochschulschriften: Reihe 36, Band 192) (zugl.: Tübingen, Univ., Diss., 1998), S. 5.

2) Ebd., S. $5 f$.

3) Bertram, Hans Georg: Material - Struktur - Form. Studien zur musikalischen Ordnung bei Johann Nepomuk David. Wiesbaden 1965.

4) Die Kirchenmusikschule wurde 1988 zur Hochschule für Kirchenmusik erhoben und zog 1998 nach Tübingen, wo sie bis heute ihren Sitz hat. Aufgrund der Erhebung zur Hochschule durfte Bertram ab 1988 den Titel "Professor an einer kirchlichen Musikhochschule" führen.

5) Haist, Bertram, S. $6 f$.

6) Mezger, Martin: Kirchenmusiker reinsten (Tauf-)Wassers. Ein Porträt des Esslinger Komponisten Hans Georg Bertram. In: Friedhelm Röttger (Hrsg.): Stiftung Esslinger Kulturpreis. Preisträger 1989-2015. Esslingen 2015, S. 24-27. 
des Projektes grob vorsortiert bereit. Ein Bericht über den aktuellen Stand und eine Anleitung zum weiteren Vorgehen bildeten dabei die Arbeitsgrundlage. Da immer wieder Anfragen von Benutzern zum Nachlass Bertram in der WLB eingingen, war die weitere detaillierte Erschließung dringend erforderlich, um den Nachlass in die Benutzung geben zu können. Zudem war die archivgerechte Verpackung und Einlagerung der einzigartigen Materialien unbedingt notwendig, um Schäden vorzubeugen. Aus diesen Gründen ergab sich für das Praxissemester im Wintersemester 2017/18 der Auftrag, die restlichen Kompositionen - also Instrumentalmusik, Bearbeitungen/Fremde Werke, Skizzen/Fragmente und Varia - zu erschließen und zu verpacken, sowie ein Nachlassverzeichnis in der Nachlassdatenbank Kalliope anzulegen. ${ }^{7}$

Bertrams kompositorisches Werk ist ausgesprochen vielfältig. Er schuf sowohl kleine Intonationen und Choralbearbeitungen für den gottesdienstlichen Gebrauch als auch abendfüllende konzertante Werke; sowohl geistliche als auch weltliche Werke, sowohl klein besetzte Kammermusik als auch orchestrale Oratorien und Konzerte. ${ }^{8}$ Dabei nahm er unterschiedlichste Einflüsse auf, entwickelte aber gleichzeitig auch seinen eigenen Stil. Bertram selbst bezeichnete sein Komponieren als eine besondere Form der Gotteserfahrung, die darin bestehe, "daß sich die Komposition wie von selbst ergibt, daß ich nicht komponiere, sondern gleichsam ,komponiert werde' oder meine, ,unter Diktat' zu schreiben. " ${ }^{9}$ Er bestreitet zwar, sich beim Komponieren theologisch zu äußern, unterstreicht aber gleichzeitig das Vertrauen, "daß Kompositionen Gotteserfahrungen vermitteln können, daß die eigene Gotteserfahrung sich dem Hörer, der Gemeinde in Kompositionen mitteilen kann. " ${ }^{10}$ Dabei verstand Bertram auch weltliche Musik "stets als Lobpreis Gottes." 11 Mit seiner Musik, egal ob weltlich oder geistlich, strebte er demzufolge danach, „Überliefertes neu zu formulieren, Brücken vom biblischen oder zumindest implizit theologischen

7) Das Nachlassverzeichnis in Kalliope ist online zu finden: Cod. hist. qt. 818. Nachlass Hans Georg Bertram, http://kalliope-verbund.info/DE-611BF-55319. Es handelt sich hierbei jedoch nur um die grobe Grundstruktur des Nachlassverzeichnisses, Details sind hier nicht enthalten.

8) Ein (unvollständiges) Werkverzeichnis auf dem Stand von 2006 findet sich online auf Bertrams Webseite, http://www.hans-georg-bertram.de/ downloads/hansgeorgbertramwerkverzeichnis.pdf.

9) Bertram, Hans Georg: Komponieren als Ausdruck von Gotteserfahrung. In: Württembergische Blätter für Kirchenmusik 55 (1988), Heft 3, S. 88-89, hier S. 89.

10) Bertram, Komponieren, S. 89.

11) Mezger, Kirchenmusiker, S. 26

12) Ebd.
Wort ins Herz heutiger Hörer zu schlagen. “"12

Trotz des "komponiert Werdens" haben Bertrams Werke oft einen langen Entstehungsprozess mit verschiedenen Entstehungsstadien, wobei nicht immer zwangsläufig alle Stadien durchlaufen werden müssen oder erhalten sind. Bertram ging planvoll und gut organisiert vor und dokumentierte sein Schaffen schon zu Lebzeiten selbst. Für die meisten seiner Werke legte er ein eigenes Inhaltsverzeichnis bei, auf dem notiert ist, welche Materialien zum Werk vorhanden sind. Notenblätter sind meist mit Datum, seinem Namen bzw. Namenskürzel und Seitenzahlen versehen, seine Schrift ist klar und gut leserlich, sodass auch aus handschriftlichen Noten musiziert werden konnte.

Am Anfang des Kompositionsprozesses steht bei Bertram häufig eine Bleistiftskizze. Manchmal handelt es sich dabei nur um einzelne Akkorde, Melodieschnipsel oder nach bestimmten Prinzipien konstruierte Tonfolgen. Oft ist in den Skizzen aber auch schon viel vom später tatsächlich verwendeten musikalischen Material erkennbar, wie etwa in jenen zu „Abendstern", einem Trio für Violine, Vi-

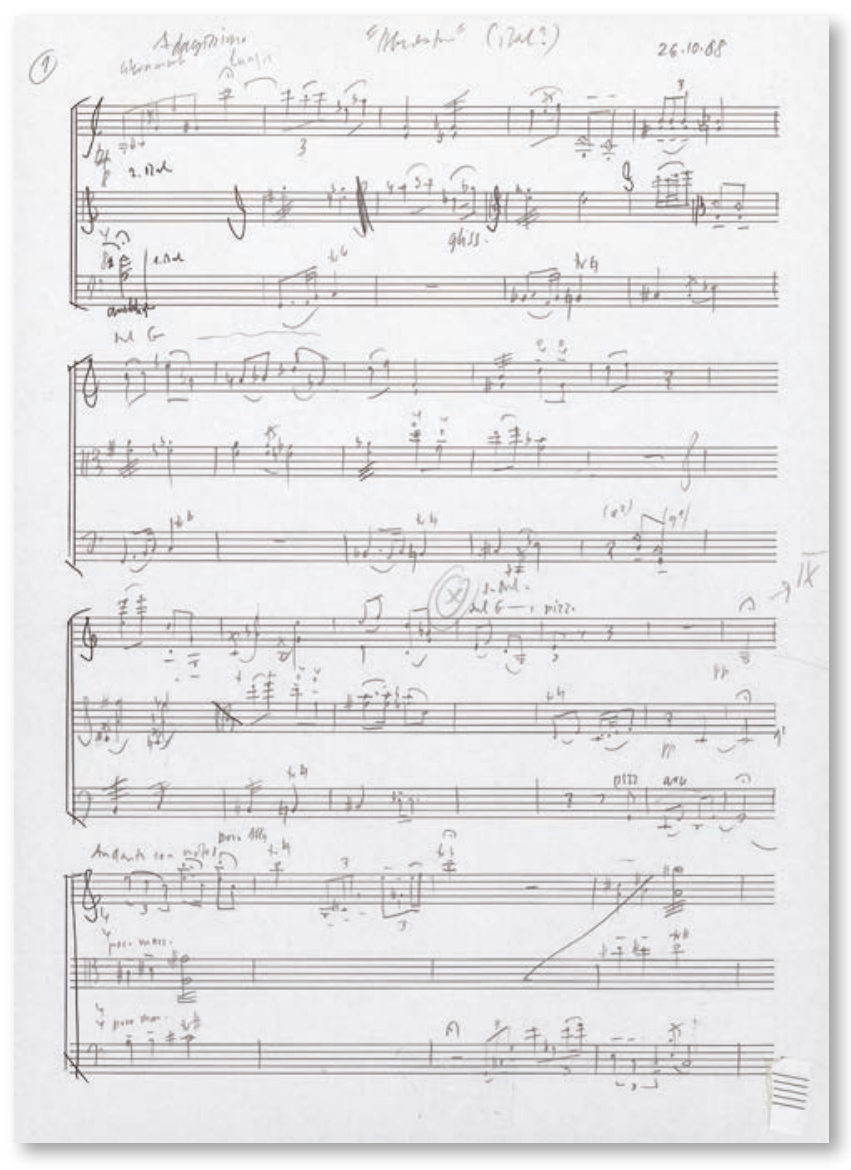

Abb. 1: Skizzenblatt zu "Abendstern" 
ola und Violoncello (Abb. 1). In den Skizzen finden sich wie hier aber häufig auch noch Streichungen, Alternativen und Korrekturen. Bertram überlegte dem Skizzenblatt zufolge auch, dem Werk einen italienischen Titel zu verleihen, was jedoch letztendlich nicht erfolgte.

Auf die Skizzen folgt meist ein so genannter Entwurf, der bereits sehr stark der fertigen Partitur ähnelt, aber noch mit Bleistift und etwas platzsparender geschrieben ist und auch noch kleinere Korrekturen enthalten kann. Die eigentliche Partitur schrieb Bertram dann sehr klar und gut leserlich, meist mit einem schwarzen Stift, wie anhand der ersten Seite der Partitur aus dem "Concerto Reale" gut erkennbar ist (Abb. 2). Für dieses Werk fertigte Bertram sogar ein eigenes Titelblatt an und gestaltete dieses mit einem grafischen Element in Kronenform. Vollendete Partituren wurden zur Vervielfältigung auf Transparentpapier übertragen und dann mittels Lichtpause vervielfältigt.

Manche Werke überarbeitete Bertram nach Fertigstellung nochmals, etwa die "Zwei Sätze über b-a$c-h$ " für vier Blechbläser. Die Partiturseite in Abb. 3

- Für Uerich Cyganek -

Hans Georg Bertram

\section{Concerto Reale}

\section{Variationen}

über das königliche Thema aus dem musikalischen Opfer

von Johann Sebastian Bach fir

Flote, Streichquartett und Cembalo

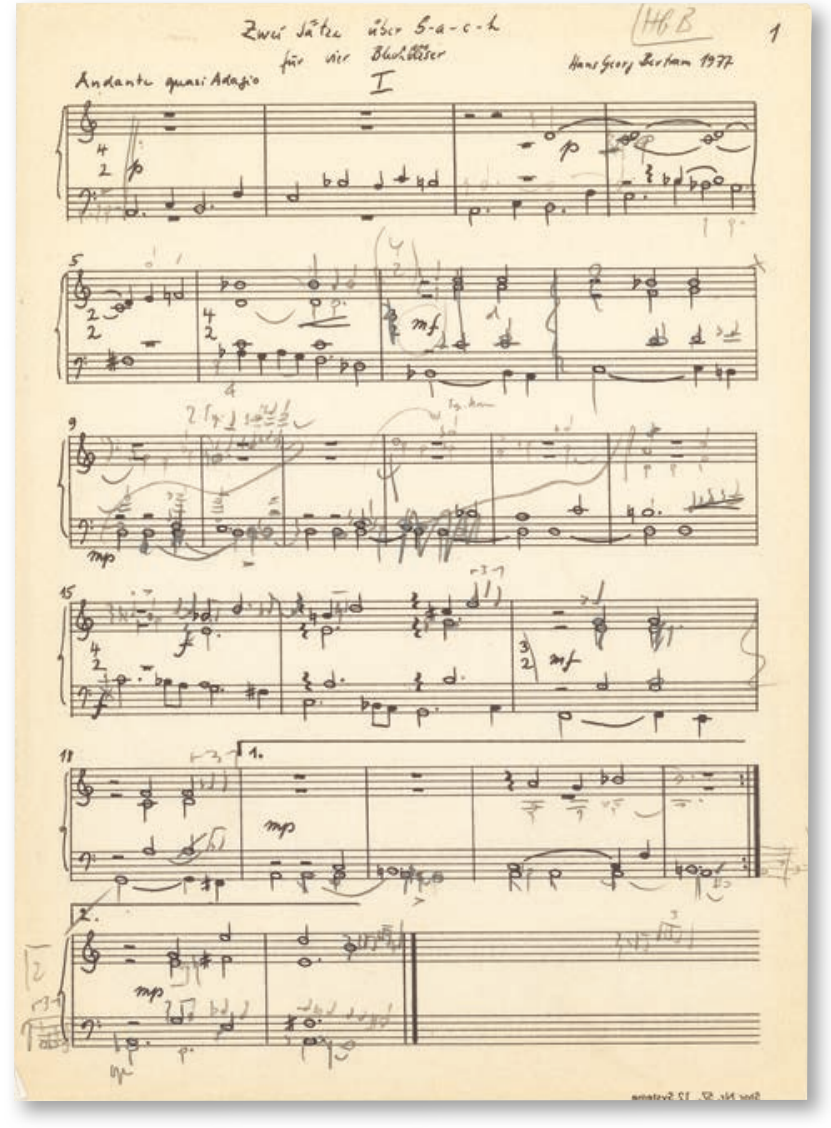

Abb. 3: Kopie der ersten Partiturseite von "Zwei Sätze über b-a-c-h" mit Skizzen zur Überarbeitung zur Zweitfassung "Du hast meine Klage verwandelt in einen Reigen"
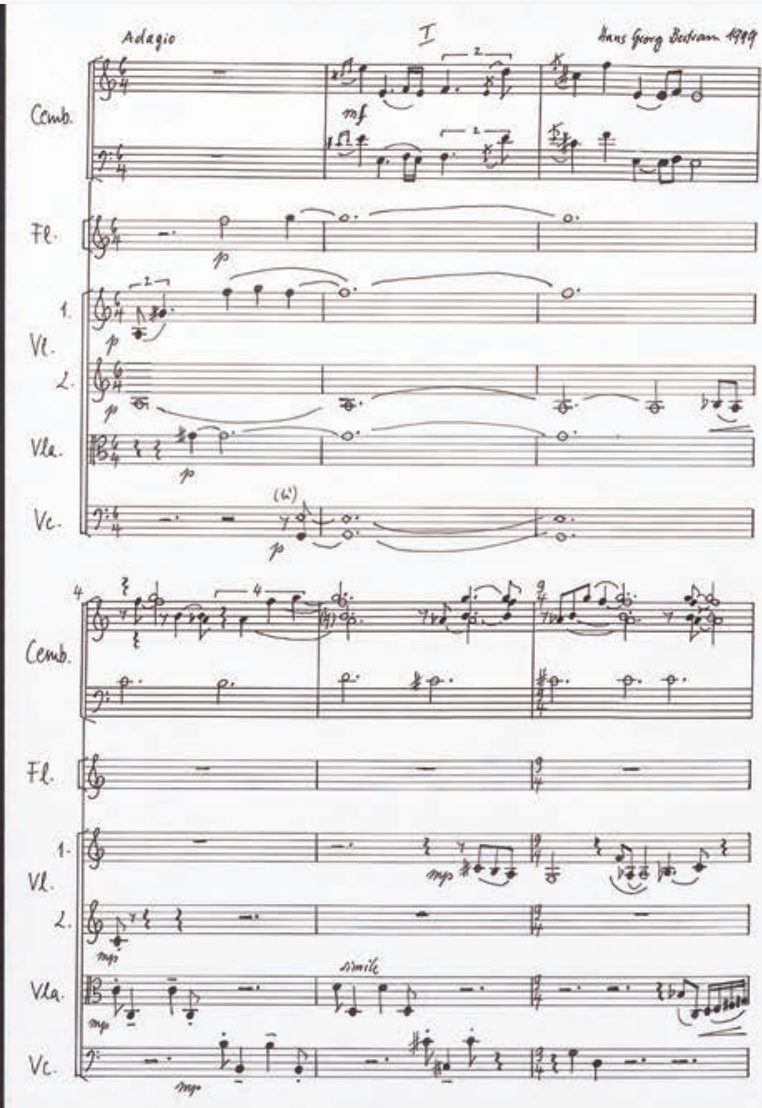
zeigt eine Kopie der Erstfassung, in die Bertram mit Bleistift die Veränderungen für die zweite Fassung skizziert hat. Die zweite Fassung erhielt später den Titel „Du hast meine Klage verwandelt in einen Reigen" und wurde im Strube-Verlag gedruckt.

Die Musik Johann Sebastian Bachs war eine wichtige Inspirationsquelle und ein Bezugspunkt für Bertram. Auch mit Werken von Wolfgang Amadeus Mozart, Johannes Brahms, Antonio Vivaldi und Georg Philipp Telemann setzte er sich kompositorisch auseinander. Dies tat er jedoch ohne Scheu vor den großen Namen und unter Verwendung verschiedenster, sogar avantgardistischer Kompositionstechniken. In diesem Sinne galt Bertram als „Konservativer, aber mit der gespannten Neugier eines Experimentators." 13

Bertram ließ sich beispielsweise auch von Anlässen in seinem Umfeld zu Kompositionen inspirieren. Zur Hochzeit von Susanne Ursula und Gerhard entwickelte er 1990 eine Tonfolge auf Grundlage der Namen des Brautpaars, wobei er den Namen der Braut größtenteils in Solmisationssilben, den Namen des Bräutigams durchgängig in Tonbuchstaben umsetzte, wie in Abb. 4 zu sehen ist. Die so entwickelte Tonfolge baute Bertram dann zur mehrsätzigen „Sonata nuptialis super nomina sponsae et sponsi pro organo" oder kurz "Sonata

super nomina" aus. Dass Bertram hier einen lateinischen Titel wählte, ist keineswegs ungewöhnlich. Auch italienische Werktitel finden sich bei inm häufig.

Anlässlich des Todes von Paul Celan 1970 komponierte Bertram die „Wandlungen“ für Orgel. Die einzelnen Sätze des Werkes tragen als Überschriften Gedichttitel Celans. Zu sehen ist in Abb. 5 die "Gauklertrommel, von meinem Herzgroschen laut". Das Werk zeugt von einer intensiven Auseinandersetzung mit Celans Lyrik. Bertram spielte es selbst auf der Orgel und mehrere Spielexemplare sind im Nachlass erhalten. Er notierte mit Bleistift und bunten Klebezetteln Fingersätze, Angaben zur Registrierung, aber auch zur künstlerischen Gestaltung. Für Musiker, die das Werk heute aufführen möchten, sind solche Spielexemplare daher eine wertvolle Quelle.

Neben solch intellektuell anspruchsvoller Auseinandersetzung lag ein weiterer Schwerpunkt von Bertrams kompositorischem Schaffen auf der Gottesdienstpraxis. So schrieb er für das 1996 neu erschienene „Evangelische Gesangbuch" Liedmelodien, etwa für EG 533 „Du kannst nicht tiefer fallen“. Doch nicht nur Melodien, auch über 200 Choralvorspiele, Orgelchoräle, Bläsersätze und Intonationen verfasste Bertram, teils für den eigenen Gebrauch, teils für Studierende oder Schüler. Eine Auswahl dieser Werke wurde als Begleitmaterial zum Evangelischen Gesangbuch gedruckt, wie etwa die Intonation zu „Du kannst nicht tiefer fallen“ (Abb. 6). Im Nachlass sind nicht nur die Reinschriften dieser Sätze erhalten, sondern auch die Verlagskorrespondenz mit Informationen zu Auswahl, Überarbeitung und Honorierung der veröffentlichten Sätze.

Durch seine Vielfältigkeit stellt der Nachlass Bertrams einen einzigartigen Fundus für Musikwissenschaftler und Musiker dar, die sich intensiver mit seinem Werk auseinandersetzen wollen. Selbst wenn die Kompositionen und Programmhefte nun vollständig detailliert

Abb. 4: Zugrundeliegende Tonfolge und erste Partiturseite der "Sonata super nomina" erschlossen sind, bleibt noch einiges zu bearbeiten: Korrespondenz, Texte, Tonträger und Dokumente. Die baldige Erschließung dieser sicherlich auch interessanten Materialien wäre durchaus 

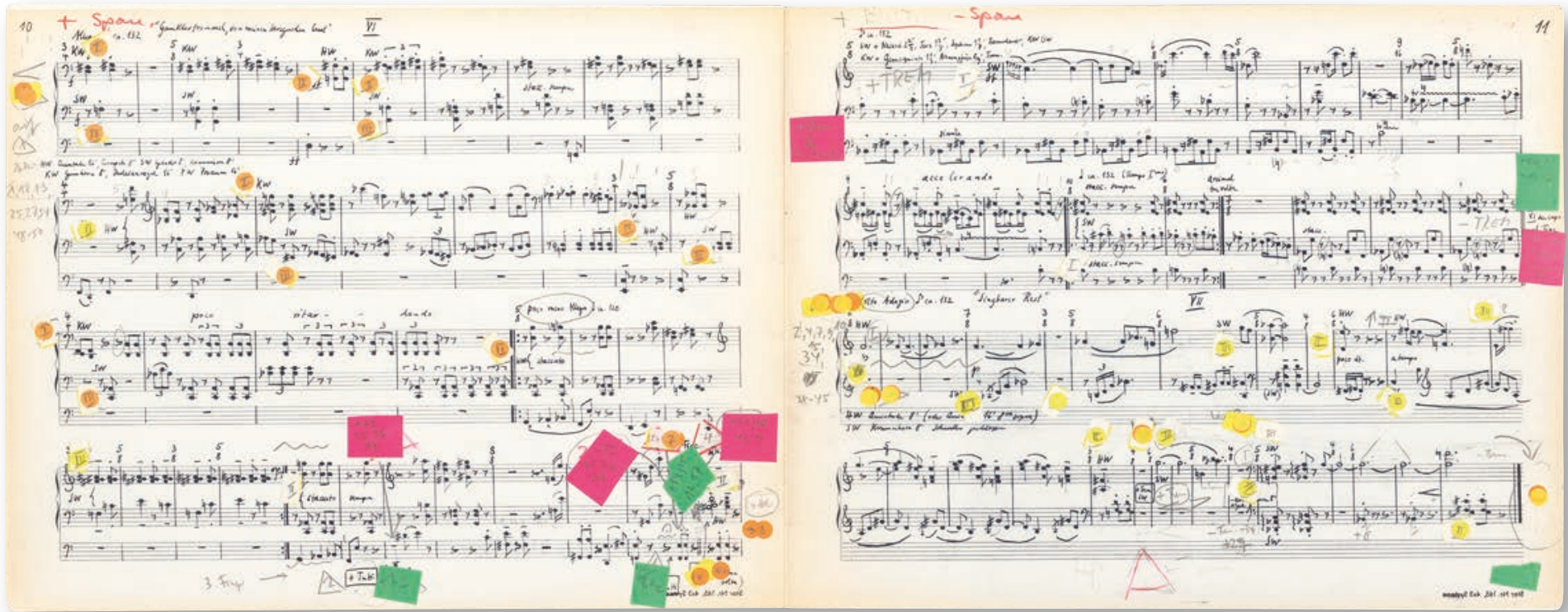

Abb. 5: Der 6. Satz der "Wandlungen", hier eines von Bertrams Spielexemplaren

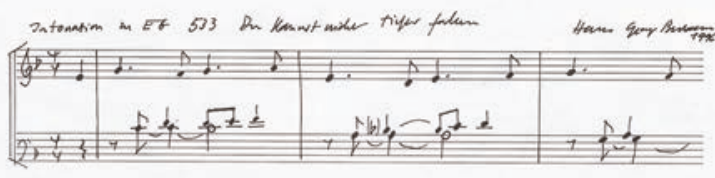

wünschenswert. Sobald der Nachlass vollständig erschlossen ist, soll das detaillierte Nachlassverzeichnis als PDF-Datei auf der Webseite der WLB veröffentlicht werden und bietet dann - ergänzend zur Nachlassübersicht in Kalliope - eine gute Re-

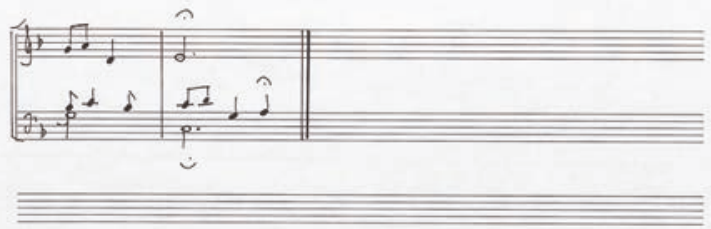
cherchemöglichkeit. So wird letztendlich Bertrams Wunsch erfüllt und der Nachlass steht der Benutzung zur Verfügung.

Anne-Marie Metzger

Abb. 6: Intonation zu "Du kannst nicht tiefer fallen" in Reinschrift 